\title{
Self-similarity in abrasion of metals by nanosharp asperities of hard carbon containing films
}

\author{
Feodor M. Borodich ${ }^{\mathrm{a})}$ \\ Technological Institute, Northwestern University, Evanston, Illinois 60208-3109 \\ Stephen J. Harris \\ Physical and Environmental Sciences Dept., MD \#3083, Ford Research Laboratory, Dearborn, \\ Michigan 48121-2053 \\ Leon M. Keer \\ Technological Institute, Northwestern University, Evanston, Illinois 60208-3109
}

(Received 3 June 2002; accepted 12 September 2002)

\begin{abstract}
We discuss a remarkably simple power-law relationship observed between the abrasion rate of an initially spherical slider by hard carbon-containing films and the number of sliding cycles $n$ to which the film has been subjected. The power-law relationship is valid up to 4 orders of magnitude in $n$. We model this phenomenon by connecting it with nanocharacteristics of the coatings. It is proposed to explain the phenomenon by a statistically self-similar variation of the pattern of relatively sharp nanometer-scale asperities of the films. (C) 2002 American Institute of Physics.
\end{abstract}

[DOI: $10.1063 / 1.1519099$ ]

The tribology of carbon-containing films such as diamondlike carbon (DLC) and boron carbide (nominally $\mathrm{B}_{4} \mathrm{C}$ ) is a subject of intensive study. ${ }^{1,2}$ While hard films are often used to reduce wear of coated components, abrasion of heavily loaded steel components such as gears by these films may, by polishing (abrading) asperities on the counterparts, enhance the fatigue resistance of the coated components by reducing a source of high intensity stresses. ${ }^{3}$ If a function of the coating is to polish the counterpart, the coating should be selected to be sufficiently abrasive to accomplish the polishing but not so abrasive as to wear away or change the geometry of the counterpart. In such cases, it may be that the abrasiveness and changes in abrasiveness may be more important to the performance of these coatings than other properties often selected for, hardness or durability. Our goal is to understand the abrasion process and the factors that control the changes in the abrasiveness of coatings.

Boron carbide is similar to amorphous, metal-containing DLC ( $a$ :Me-DLC) in the sense that they are both hard, amorphous, carbon-containing films with large amounts of other elements. Both coatings can substantially reduce the roughness of a steel counterpart (for example, from 250 to $50 \mathrm{~nm}$ within less than 100 cycles) through a mechanical polishing process. The abrasiveness of a boron carbide or DLC film actually drops rapidly as it polishes the steel, coinciding with the loss of relatively sharp nanometer-scale film asperities as observed in scanning electron microscopy (SEM) images (Fig. 1). The morphology of the coating on a scale larger than a few microns is not correlated with the abrasion rate. ${ }^{4}$ This counterpolishing of the coating by the steel appears to be at least partly chemical in nature. ${ }^{2,5}$ In this letter, we describe a model to describe the kinetics of the counterpolishing process based on statistical and similarity methods. ${ }^{6}$

The average abrasion rate is defined as the total volume

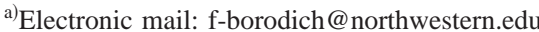

of steel removed $M$ divided by the total distance traveled by the ball $d=2 \pi R n$ with a pin-on-disk radius $R$; or as the average of the instantaneous abrasion rates $A_{i}$ (abrasion rate on the $i$ th cycle) during the first $n$ cycles,

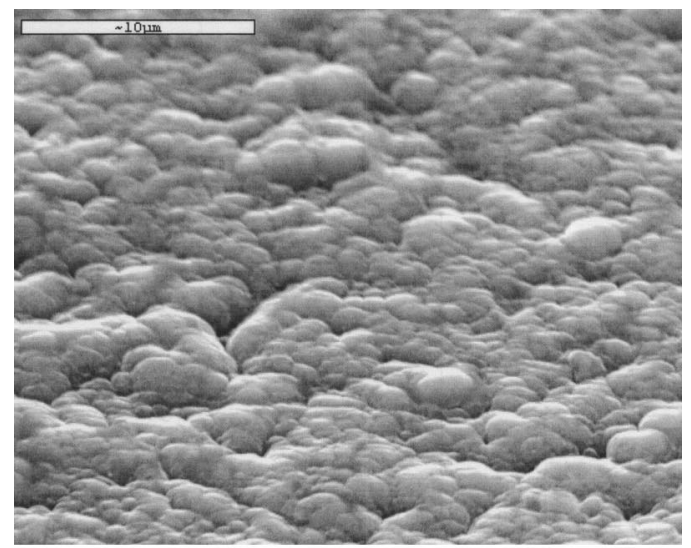

(a)

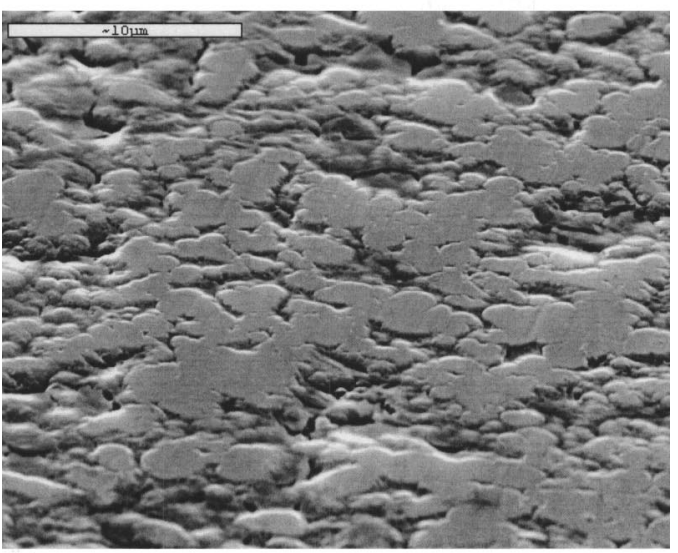

(b)

FIG. 1. SEM images of a $\mathrm{B}_{4} \mathrm{C}$ film $(30 \times 30 \mu \mathrm{m}$ region). (a) Unworn. (b) After 500 cycles. 


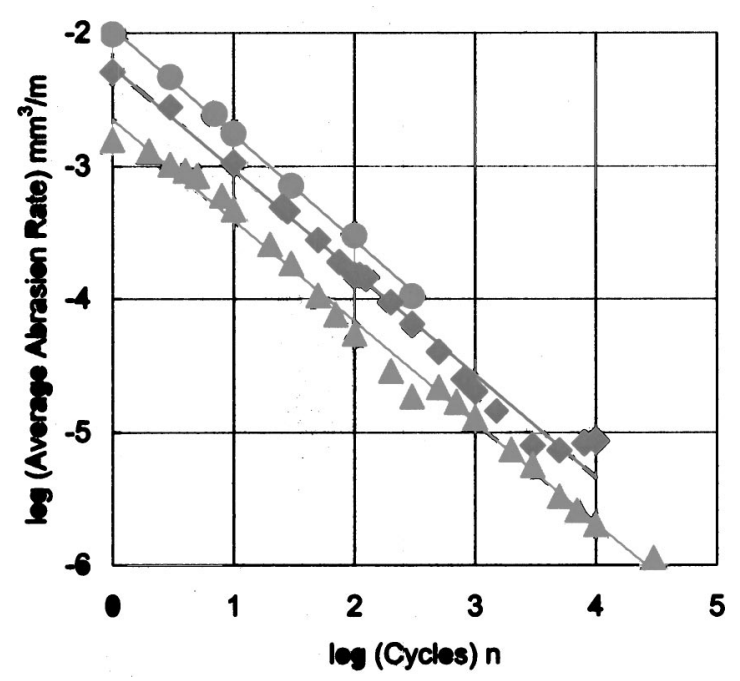

FIG. 2. Abrasion kinetics of $\mathrm{B}_{4} \mathrm{C}$ for loads of 1 (triangles), 5 (squares), and $11 \mathrm{~N}$ (circles).

$$
\overline{A(n)}=\frac{M}{d}=\frac{1}{n} \sum_{i=1}^{n} A_{i} .
$$

$M$ is determined from three-dimensional optical profilometer images of wear scars on the steel balls, calculating the volume removed by numerical integration of the volume under the surface of the worn ball ${ }^{7}$ and subtracting it from the volume of a perfect sphere. For a fixed load, we have shown that the abrasion rate depends only on properties of the film and is constant during a cycle. ${ }^{4,8}$

Because abrasion is controlled by details of the local morphology and pressure, Fig. 1 suggests that there should be a very complex time history for the abrasion rate. In particular, it would be surprising if the abrasion rate caused by the coating surface of Fig. 1(b) could be simply related to or predicted by the abrasion rate caused by the coating surface of Fig. 1(a), especially since the steel surface also changes. Nevertheless, the variation of $\overline{A(n)}$ follows an extraordinarily simple history for up to $10^{4.5}$ cycles (Figs. 2 and 3 ),

$$
\overline{A(n)}=A_{1} n^{\beta}
$$

where $\beta$ is an experimental parameter that must lie within the range $4-1 \leqslant \beta \leqslant 0$ and is typically -0.7 to -0.8 for both $\mathrm{B}_{4} \mathrm{C}$ and DLC films, independent of hardness (8 to $\left.23 \mathrm{GPa}\right)$ of the film. It is straightforward to derive from Eq. (1) and

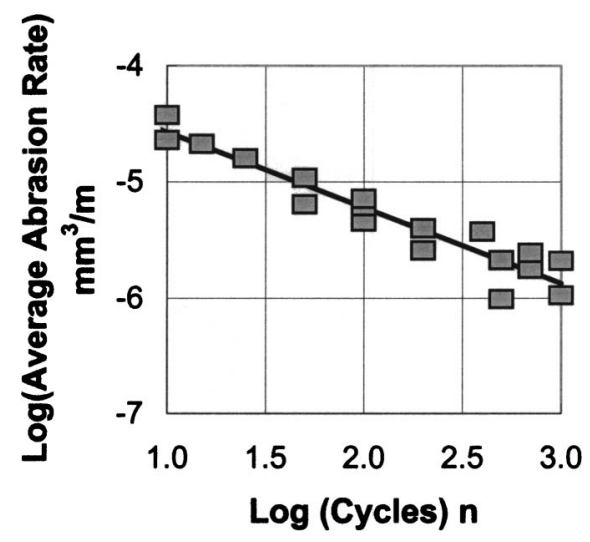

FIG. 3. Abrasion kinetics of a:Me-DLC for a load of $5 \mathrm{~N}$.

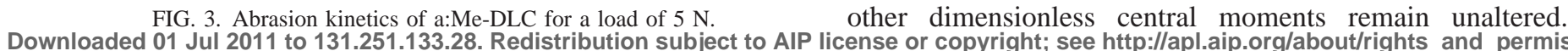

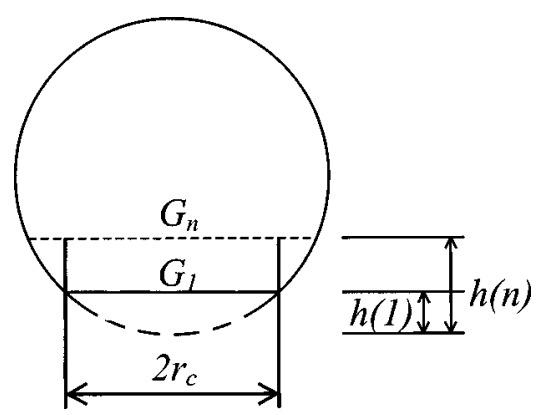

FIG. 4. The total volume removed from the ball and the wear governing cylinder.

Eq. (2) that $A_{i} \cong(1+\beta) A_{1} i^{\beta}$ with an error of less than $1 \%$ if $\beta$ is -0.8 and $i \geqslant 6$. Apparently, once $\beta$ is known, the abrasiveness of the film of Fig. 1(b) can indeed be predicted knowing just $A_{1}$, the abrasiveness of the film of Fig. 1(a). A relationship as simple, accurate, and widely applicable as Eq. (2) is rarely observed in the study of wear.

We model this phenomenon by connecting it with nanocharacteristics of the coatings. We assume that (1) the abrasion is due to sharp asperities; (2) the loss of abrasiveness is assumed due to the evolution of sharp asperities to flatter structures, as seen in Fig. 1; (3) the surface of the coating can be described in a statistical way in terms of the distance $\ell$ between sharp asperities (see next); and (4) the abrasiveness of the coating is proportional to number of asperities which, for a fixed coating area, means that the abrasiveness in proportional to $\ell^{-2}$, the asperity density.

Consider a steel ball which is undergoing abrasion by sliding against a coated coupon. We take the period of the first cycle $T$ as the unit of time. So, we can use the number of cycles $n$ as time instead of $t=n T$. After the first cycle, enough material has been worn away so that the edge of the ball has receded by a distance $h(1)$, creating a flat wear region on the ball that we will call $G_{1}$ (see Fig. 4). The ball continues to wear until after $n$ cycles the edge has receded by a distance $h(n)$, creating a larger flat wear region on the ball which we call $G_{n}$. Since the rate of recession of the steel ball $d h / d n$ must be the same everywhere in $G_{n}$, the overall recession rate can not exceed the recession rate of the least abrasive subregion within $G_{n}$. And since the abrasion rate falls monotonically with $n$, that subregion is the one that has been in contact longest, i.e., $G_{1}$. Therefore, to model changes in abrasiveness with time, it is only necessary to model changes in abrasiveness in the central region $G_{1}$. That is, the part of the coating that is in contact with the subregion $G_{1}$ is less abrasive than any other part of the coating.

Consider a set of random points on the coating that is in contact with the central region $G_{1}$. These points comprise the peaks of sharp asperities on the coating whose average separation distance is $\ell$. Sets of such points, distributed in an irregular way within a planar region, are known to arise in many disciplines. ${ }^{9-11}$ We assume that the pattern of sharp asperities within the region in contact with $G_{1}$ can be described in a statistically self-similar way, ${ }^{6,12}$ which means that the distribution of the points of sharp asperity peaks within the region is independent of $n$; i.e., only the mean $\ell$ of the probability distribution changes its value while all license or copyright; see http://apl.aip.org/about/rights_and_permissions 
Hence, the images of the pattern after any number of cycles $n_{1}$ and $n_{2}$ can not be distinguished statistically from each other if the average distance $\ell(n)$ between the points is not known. For such a steady-state process, we can write $\ell\left(n_{1}\right) / \ell\left(n_{2}\right)=f\left(n_{1} / n_{2}\right)$. Similarly, we obtain $\ell\left(n_{1}\right) / \ell\left(n_{3}\right)$ $=f\left(n_{1} / n_{3}\right)$ and $\ell\left(n_{2}\right) / \ell\left(n_{3}\right)=f\left(n_{2} / n_{3}\right)$. If we denote $x$ $=n_{1} / n_{3}$ and $y=n_{1} / n_{2}$ then we can write ${ }^{6,12} f(x) / f(y)$ $=f(x / y)$. This equation is satisfied only by $f(x)=x^{\alpha}, \alpha$ $=f^{\prime}(1)$. Thus, this relates $f(x)$ to $\ell(n)$ and we see that $\ell(n)=\ell\left(n_{1}\right)\left(n / n_{1}\right)^{\alpha}, \alpha>0$. If the average spacing $\ell(n)$ $=\sqrt{\left|G_{1}\right| / N(n)}$, where $N(n)$ is the number of sharp asperities within the area $\left|G_{1}\right|$ after $n$ cycles, we obtain $N(n)$ $=N(1) n^{-2 \alpha}$. The volume of steel $M_{c}=\left|G_{1}\right| h(n)$ removed from the region of the coating in contact with $G_{1}$ during the first $n$ cycles is

$$
M_{c}=\sum_{i=1}^{n} M_{c i}=\sum_{i=1}^{n} m N(i)=\sum_{i=1}^{n} M_{c 1} i^{-2 \alpha},
$$

where $m$ is the average amount of material on the steel ball removed by a sharp asperity during a cycle, $M_{c 1}=m N(1)$, $M_{c i}$ is the volume of steel removed from the central part $G_{1}$ on the $i$ th cycle. Approximating the sum by an integral, we obtain $h(n) \approx \int_{0}^{n} h(x) d x \approx h(1) n^{1-2 \alpha}$.

In the Hertzian approximation, a ball is described as $z$ $=\left(1 / 2 R_{b}\right) r^{2}$ where $R_{b}$ is the ball radius and $z, r$ are cylindrical coordinates. The volume of the Hertzian ball under the planar cross section at the height $h$ is $V(h)=\pi R_{b} h^{2}$. The material removed from the ball is governed by the cylinder of approximate radius $r_{c} \cong \sqrt{\left|G_{1}\right| / \pi}$ and the height $h(n)$ (Fig. 4). So, the total volume of steel $M \equiv V(h)$ removed from the slider during $n$ cycles can be approximated $V(h(n))=\pi R_{b} h^{2}(n) \approx \pi R_{b} h^{2}(1) n^{2(1-2 \alpha)} \quad$ and $\overline{A(n)}$ $\approx \overline{A(1)} n^{2(1-2 \alpha)} / n$ which, for $\beta=1-4 \alpha$, yields Eq. (2).

Obviously, the surfaces in Fig. 1 do not transform into each other upon dilation. Nevertheless, our analysis shows that if the sharp asperities remain randomly distributed in the sense discussed herein, then the distribution function for the distance between the asperities is self-similar and the abrasion law Eq. (2) follows. It is interesting to note that if the probability that any asperity loses its sharpness were independent of $n$, we would have $\ell\left(n_{1}\right) / \ell\left(n_{2}\right)=f\left(n_{2}-n_{1}\right)$, and Figs. 2 and 3 would show exponential behavior. Power-law behavior requires that the probability for an asperity to lose its sharpness falls inversely with $n$, a stringent constraint on the physics of the process. Perhaps the only asperities that remain sharp after many cycles are those that are protected by neighboring structures. What such protection means and why there is precisely an inverse relation with $n$ is being studied. Insight from this work will promote development of coatings with properties optimized specifically to protect against fatigue.

${ }^{1}$ A. Erdemir, in Modern Tribology Handbook, edited by B. Bhushan (CRC Press, Boca Raton, 2001), Vol. 2, pp. 871-908.

${ }^{2}$ S. J. Harris, G. G. Krauss, S. J. Simko, R. J. Baird, S. A. Gebremariam, and G. Doll, Wear 252, 161 (2002).

${ }^{3}$ T. L. Krantz, M. P. Alanou, H. P. Evans, and R. W. Snidle, J. Tribol. 123, 709 (2001).

${ }^{4}$ S. J. Harris and A. M. Weiner, Wear 223, 31 (1998).

${ }^{5}$ Y. G. Gogotsi, A. M. Koval'chenko, and I. A. Kossko, Wear 154, 133 (1992).

${ }^{6}$ G. I. Barenblatt, Scaling, Self-Similarity, and Intermediate Asymptotics (Cambridge University Press, Cambridge, UK, 1996).

${ }^{7}$ S. J. Harris and G. G. Krauss, Tribol. Lett. 10, 187 (2001).

${ }^{8}$ S. J. Harris and A. M. Weiner, Wear 213, 200 (1997).

${ }^{9}$ R. N. Bracewell, Two-Dimensional Imaging (Prentice Hall, Englewood Cliffs, NJ, 1995).

${ }^{10}$ P. J. Diggle, Statistical Analysis of Spatial Point Patterns (Academic Press, London, 1983).

${ }^{11}$ E. S. Venttsel and L. A. Ovcharov, Theory of Probability (Nauka, Moscow, 1973).

${ }^{12}$ F. M. Borodich, Mechanics of Soilds (Allerton Press, Inc. New York, 1986). 